\title{
Un modelo de la percepción de calidad en servicios puros por Internet Aplicación al sistema de inscripciones en línea en una institución educativa ${ }^{1}$
}

\section{Mila María Gamboa García Brand Developer Knorr, LATAM UNILEVER mila.gamboa@gmail.com \\ Rogerio Domenge \\ Muñoz \\ Instituto Tecnológico Autóno- mo de México domenge@itam.mx}

${ }^{1}$ Se agradece a la Asociación Mexicana de Cultura, A.C. por el apoyo en la elaboración de la presente investigación.

\section{Resumen}

Debido a la creciente importancia del uso de Internet como medio de servicio en diferentes tipos de organizaciones, la percepción de su calidad ha resultado ser cada vez más importante como elemento indispensable en su diseño y rediseño para lograr satisfacer las expectativas de sus usuarios. El objetivo de este artículo es presentar y validar un modelo de la percepción de la calidad en servicios puros por Internet, así como identificar sus impactos. La investigación se basa en modelos de ecuaciones estructurales. Los resultados sugieren que la percepción de la calidad tiene una relación positiva con la intención de continuar utilizando y recomendando el servicio. Los usuarios consideran la eficiencia, la confiabilidad, la disponibilidad y la privacidad del servicio como factores significativos en su percepción de calidad. No obstante, cuando el usuario enfrenta problemas con el servicio, también considera la posibilidad de corregir errores, la capacidad de respuesta del sitio y la existencia y facilidad de asistencia personal.

Palabras clave: calidad en el servicio, Internet, modelos ecuaciones estructurales. 


\title{
A quality perception model for on-line pure services: application in an on-line registration system of an academic institution
}

\begin{abstract}
As Internet use for service purposes has become increasingly important in many types of organizations, customer perception of its quality has become a fundamental criterion for designing and redesigning these services to satisfy customer expectations. The objective of this article is to propose and test a model for measuring quality perception in a pure Internet services as well as identifying the impacts of the quality perceptions. This research is based on structural equations modeling. The results suggest that quality perception has a positive relation to the user's intention of re-using and recommending the service. Users consider efficiency, reliability, availability, and privacy as significant factors in their service quality perception. Nevertheless, when the user has problems with the service, he also evaluates the possibility of correcting errors, the site's response capability, and the existence and availability of personal customer service.
\end{abstract}

Keywords: service quality, Internet, structural equation modeling.

\section{Introducción}

Cada vez resulta más importante para una organización de servicios la percepción que tienen sus usuarios o clientes de la calidad en el servicio que ofrece. Si la organización tiene una clara orientación al mercado y se preocupa por las características de la demanda de sus clientes o usuarios, incorporándolas en el diseño y rediseño del servicio que ofrece, la probabilidad de lograr sus objetivos será mayor, lo cual se reflejará finalmente en una mayor intensidad de uso del servicio y en la intención de continuar utilizándolo en el futuro aunado a un incremento en las posibilidades de ser recomendado a usuarios potenciales. Si los usuarios perciben un alto nivel de calidad en el servicio, éste tendrá un alto valor para ellos, lo cual es uno de los fundamentos de la organización de servicios: ofrecer valor a sus usuarios, ya sean servicios tradicionales (ST) o servicios por Internet (SI).

En particular, el rediseño de un si debería ser una actividad cotidiana en toda empresa orientada al mercado. Las necesidades actuales de los usuarios de si cambian constante y rápidamente, debido principalmente a los avances en la tecnología y a cambios en sus necesidades, gustos y modas. 
La identificación de los factores que los clientes consideran como importantes en su percepción de la calidad del servicio sienta la base para definir las características ideales que deberá tener tanto el servicio a ofrecer en sí mismo como el sistema operativo que lo hace posible y lo pone a la disposición del cliente. A partir de las diferencias que pudieran existir entre las características ideales y las reales de un sistema ya existente, se podrán determinar las áreas de oportunidad para mejorarlo.

\section{Antecedentes teóricos}

Algunos autores han identificado a la calidad en el servicio como un tipo de actitud, relacionada, pero no equivalente a la satisfacción (Bolton y Drew, 1991; Parasuraman et al., 1985). Cronin y Taylor (1994) marcan una clara distinción entre calidad y satisfacción, considerando a la primera como un tipo de actitud que se forma a partir de una evaluación de largo plazo, mientras que la satisfacción es una medida específica para cada transacción. Ruyter et al. (1997) consideran a la calidad como un antecedente de la satisfacción y señala al desempeño del servicio como el elemento determinante en la percepción de calidad.

En el contexto de los servicios, la calidad es un concepto difícil de definir porque es percibida por el cliente a través de numerosos dimensiones o factores. Debido a que no existen factores universales que la determinen, numerosos autores han desarrollado sus propios modelos de calidad en el servicio.

\section{Modelos de calidad en el servicio tradicional}

Múltiples investigaciones sugieren que, en lugar de percibir la calidad de manera unifactorial, los consumidores forman su percepción de calidad mediante diversos factores de acuerdo con el contexto del servicio (Brady y Cronin, 2001; Zeithaml et al., 2006). Sasser et al. (1978), pioneros en el tema de calidad en el servicio, consideran que dicha calidad es más compleja y difícil de evaluar que la calidad en los productos, donde la percepción juega un aspecto fundamental. La percepción es una etapa del procesamiento de información que incluye la exposición a un estímulo, la atención que presta el individuo al estímulo y la interpretación que se le da al mismo (Hawkins et al., 2004).

Swan y Combs (1976) definen al servicio percibido como el resultado de un proceso de evaluación de la experiencia del consumidor que puede ser subdividido, a 
su vez, en dos procesos: desempeño instrumental o técnico y desempeño expresivo o nivel de satisfacción. Con base en este concepto, hacen una distinción entre dos factores fundamentales de la calidad del servicio percibida: calidad técnica - resultado que el consumidor recibe como consecuencia del servicio y que en ocasiones puede ser medida de manera objetiva; es una dimensión necesaria, mas no suficiente, para la satisfacción del cliente - y calidad funcional (manera en que se desarrolla la prestación del servicio).

Grönroos (1982) desarrolló un modelo de calidad en servicios bajo el concepto de que la calidad es resultado de la comparación entre el servicio esperado y el servicio percibido. El modelo de Grönroos (1982) se distingue como uno de los precursores de la corriente europea del análisis de calidad en el servicio. Kang y James (2004) consideran que este modelo tiene la ventaja conceptual con respecto a sus contrapartes estadounidenses, en su mayoría basadas en el modelo de Parasuraman et al. (1985) de analizar la calidad en el servicio desde un punto de vista más holístico, pues además de incluir la perspectiva técnica incluye la perspectiva funcional y la imagen percibida.

Asimismo, Grönroos (1982), U. Lehtinen y J. Lehtinen (1982) desarrollaron un modelo que identifica un conjunto de brechas, discrepancias o deficiencias (gaps) existentes con respecto a las percepciones de los directivos de calidad en el servicio y las tareas asociadas a la prestación del servicio al consumidor. Estas deficiencias pueden ser obstáculos al ofrecer un servicio que sea percibido por el consumidor como de alta calidad (Parasuraman et al., 1985), considerando las discrepancias entre lo que la administración cree que los compradores quieren y lo que los clientes realmente quieren o demandan, lo cual moldea sus expectativas de servicio a partir de sus necesidades, sus experiencias previas, la comunicación verbal de persona a persona que ha recibido y las comunicaciones por parte del prestador de servicios, Al recibir el servicio, se genera en el cliente una comparación o una percepción relativa en función de su expectativa de nivel de calidad que éste debería tener.

\section{Modelos de calidad en el servicio por Internet}

Los servicios electrónicos o por Internet presentan características que los distinguen de los demás servicios: una mínima o nula interacción humana, un diseño muy preciso del servicio y un canal de comunicación de tipo electrónico. Parasuraman et al. (2005) clasifican a los SI en servicios puros y no puros. El propósito 
principal en un servicio puro es otorgar un beneficio de tipo no material al consumidor (servicio financiero, información, etc.); en cambio, en un servicio no puro, la actividad principal se orienta a la venta de bienes a través de Internet.

De acuerdo con Zeithaml et al. (2006), los servicios por Internet generan beneficios y retos distintos a los servicios ofrecidos por canales no electrónicos (estandarización, bajo costo, conveniencia para el cliente, amplio acceso y mayor distribución). La calidad en servicios tradicionales ha sido estudiada considerando que el factor humano es crítico (Zeithaml et al., 2000); en consecuencia, es aún incierto que las mismas dimensiones que han sido identificadas como factores de evaluación de calidad en estos servicios sean las mismas que para servicios donde el contacto personal es mínimo o nulo, tal como sucede en los Si. Loiacono et al. (2002) consideran tres elementos que se distinguen en la evaluación de la calidad de un sitio de Internet: máquina (un software analiza automáticamente las características del sitio), juicio de expertos (identifican criterios de evaluación para la calidad de sitios de una misma categoría o función) y usuario final (se pregunta al visitante del sitio su opinión sobre el sitio).

Las empresas consultoras y prestadoras de sI han desarrollado mediciones propias de calidad en el servicio de acuerdo con sus necesidades específicas. En la mayoría de los casos, sus resultados son poco generalizables hacia otros si porque no existe una conceptualización formal de los elementos formadores de la calidad y su operacionalización generalmente no sigue un desarrollo formal. Los enfoques de este tipo de investigación son muy diversos: desde encuestar a los Webmasters de sitios de empresas Fortune 1000 sobre los factores más importantes de éxito de sus portales (Zeithaml et al., 2002), hasta el desarrollo de métodos y escalas propias para evaluación directa por parte del consumidor.

Si bien en los sitios de Internet se pueden identificar características comunes, resulta de utilidad realizar una distinción de acuerdo con su función práctica o comercial porque ésta puede variar significativamente. Desde esta perspectiva, es posible identificar cinco tipos básicos de sitios por Internet (Informaticamilenium. com.mx): sitios de negocios, que tienen como objetivo principal dar a conocer a la empresa y sus productos; sitios de servicio que ofrecen un servicio a sus clientes, proveedores y demás socios comerciales (bases de datos, pedidos, noticias, etc.); sitios de comercio electrónico o tiendas virtuales; sitios de imagen corporativa, que tienen como propósito principal comunicar la imagen de la compañía al mercado para reforzar su posicionamiento y sitios de entretenimiento. Cada uno de 
estos sitios tiene características propias, sin embargo, con el objetivo de evaluar su calidad, todos ellos están orientados a satisfacer las necesidades del usuario de la manera más adecuada incluyendo principalmente su utilidad, eficacia, interacción, accesibilidad, alcance, desempeño y servicio.

Por otro lado, los esfuerzos académicos para la medición de calidad en si están caracterizados por tener un mayor rigor en la conceptualización y operacionalización de los factores relevantes. Existen seis factores, recurrentes en varias investigaciones, mediante los cuales los clientes evalúan la calidad en si: información y contenido, facilidad de uso, privacidad y seguridad, diseño del sitio, satisfacción y confiabilidad y otros criterios (tales como facilidad de acceso, personalización del sitio y capacidad de respuesta). Es importante considerar que en las transacciones por Internet, el consumidor generalmente está muy orientado al objetivo (goaloriented), lo cual ocasiona que otros factores no sean relevantes (Zeithaml et al., 2002).

Se han desarrollado varios modelos de calidad en si y sus correspondientes instrumentos de medición. El instrumento WebQualtm (Loiacono et al., 2002) fue desarrollado con el objetivo de medir la percepción de calidad de los sitios en Internet que realizan transacciones con el consumidor final (business to consumer). El modelo de brechas (gaps) de Parasuraman et al. (1985) refleja posibles deficiencias ocasionadas por los prestadores de servicio que contribuyen a una percepción de calidad deficiente. Zeithaml et al. (2002) consideran posible extender este modelo a los SI, considerando cuatro brechas: información, diseño, comunicación y satisfacción. El instrumento WEBQUAL de Fransi (2003) empleó el modelo conceptual de Grönroos para formar un cuestionario que midiera las dimensiones de diseño del establecimiento virtual, comercialización de producto y servicios a través de la red, calidad del servicio prestado al cliente y los determinantes del comportamiento del consumidor en línea.

El instrumento E-SQual de Parasuraman et al. (2005) se basa en el enfoque de la cadena de medios finales, utilizada para comprender la estructura cognitiva del consumidor. La eficiencia, el cumplimiento, la confiabilidad y la privacidad se consideran relevantes en todo tipo de servicios y se reflejan en el instrumento E-SQual. La capacidad de respuesta, la indemnización y el contacto fueron identificados como sobresalientes sólo cuando existen preguntas sobre el servicio o problemas al utilizarlo. Para analizarlas, los autores crearon una escala separada a la que nombraron E-RecSQual. Adicionalmente, Parasuraman et al. (2005) inclu- 
yeron dos factores que son relevantes para dimensionar la calidad en los si: el valor percibido y las intenciones de fidelidad al servicio evaluado. Finalmente, incluyeron dos medidas globales de calidad y valor percibido del servicio, así como una sección de características demográficas y de uso de sI.

\section{Modelo propuesto de percepción de calidad en Servicios por Internet y plan- teamiento de hipótesis}

Las hipótesis de esta investigación tienen como objetivo general identificar el impacto de la percepción de la calidad en servicios por internet en el valor y la intención de uso y recomendación. Para tal fin se plantea un modelo global (figura 1), formulado a partir de las relaciones planteadas por Parasuraman et al. (2005). Los factores que definen la percepción de calidad en si pueden ser divididas en dos grupos. En el primer grupo se encuentran cuatro dimensiones utilizadas para medir la percepción del servicio de cualquier usuario de un sI (eficiencia, disponibilidad del sistema, confiabilidad y privacidad). En el segundo grupo (sombreado en la figura 1) se encuentran los factores que los autores identifican como relevantes cuando existe algún problema o contingencia con el servicio (capacidad de respuesta, indemnización y contacto). Adicionalmente, se muestra la relación entre calidad global, valor percibido e intenciones de permanecer y recomendar el si que son propuestas por los autores como consecuencias de la evaluación de la calidad en el SI. Las variables latentes (círculos) y las observadas (rectángulos) consideradas en el modelo se especifican en el Anexo 1.

Derivados del modelo global se especifican cinco modelos parciales (cuadro 1) con el objetivo de realizar diversos análisis más específicos, considerando la complejidad en la identificación del modelo global.

Las cuatro hipótesis que se plantean se basan en la proposición de que las consecuencias de una percepción más alta en la calidad en el si tenderán a influir positivamente tanto en el valor percibido como en la intención de utilizar y recomendar el SI.

H1: La percepción de la calidad en el servicio normal tiene una relación positiva con la percepción de calidad (global).

H2: La percepción de la calidad en el servicio de contingencia tiene una relación positiva con la percepción de calidad (global). 


\section{Figura 1}

Modelo global de percepción de calidad de sI y sus consecuencias Dimensiones de la calidad en si puros y su relación con la calidad, valor agregado $\mathrm{e}$ intenciones de permanecer $\mathrm{y}$ recomendar el servicio

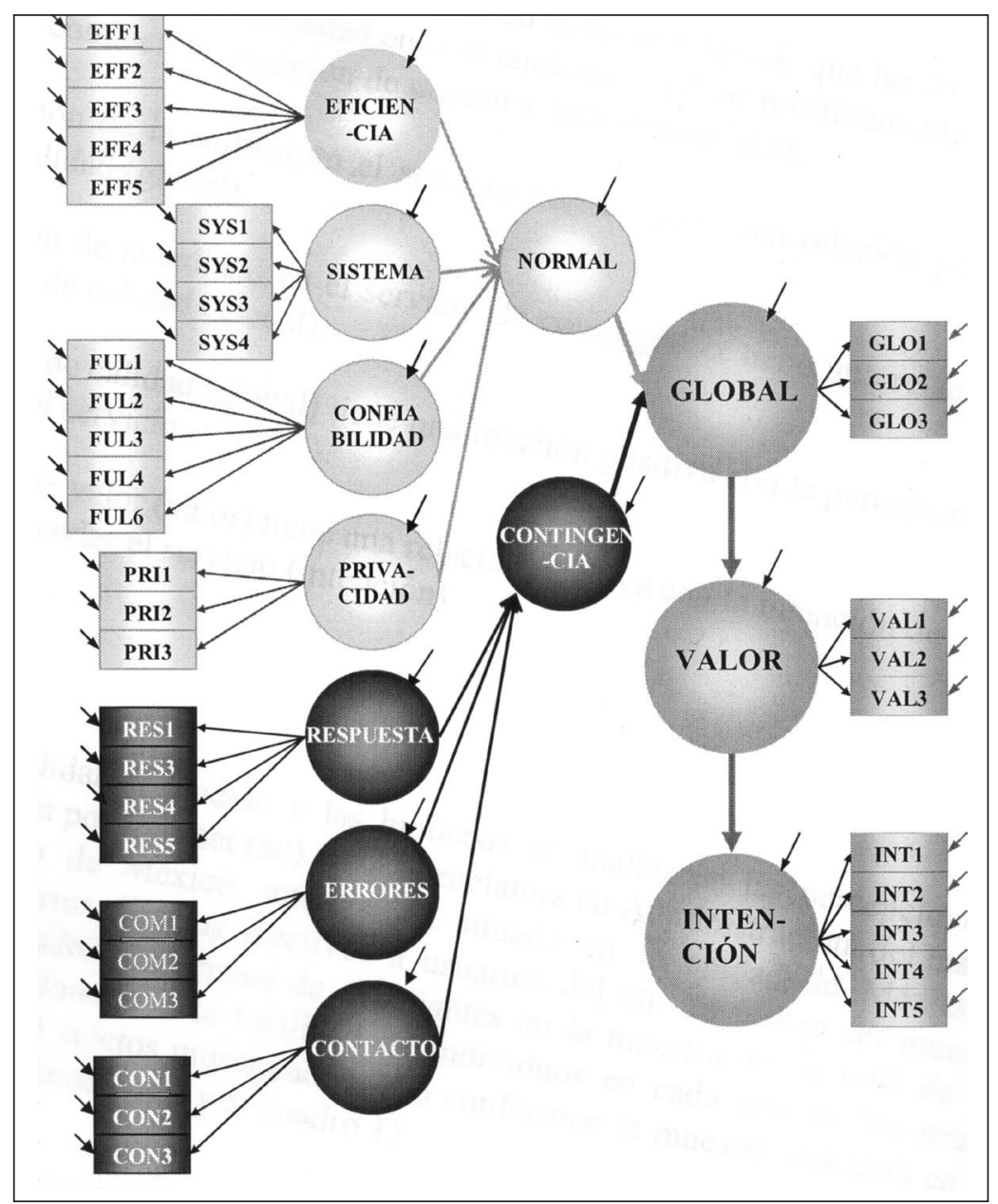

Fuente: Basado en Zeithaml et al., 2002 y Parasuraman et al., 2005. 


\section{Cuadro 1}

\section{Resumen de las características de los modelos parciales}

\begin{tabular}{c|c|c|c|c}
\hline \multicolumn{5}{c}{ Características de los modelos parciales } \\
\hline \multicolumn{2}{c}{ Dimensiones consideradas } & $\begin{array}{c}\text { Análisis de la percepción de calidad } \\
\text { global y valor agregado }\end{array}$ & $\begin{array}{c}\text { Tamaño de } \\
\text { muestra }\end{array}$ \\
\hline & Normal & Contingencia & Junta: gloval & 453 \\
Modelo 1 & $\checkmark$ & $\checkmark$ & Separada: global-valor & 453 \\
Modelo 2 & $\checkmark$ & $\mathbf{x}$ & Junta: gloval & 453 \\
Modelo 3 & $\checkmark$ & $\mathbf{x}$ & Separada: global-valor & 176 \\
Modelo 4 & $\mathbf{x}$ & $\checkmark$ & Junta: gloval & 176 \\
Modelo 5 & $\mathbf{x}$ & $\checkmark$ & & \\
\hline
\end{tabular}

H3: La percepción de calidad (global) tiene una relación positiva con la percepción de valor agregado (valor) del servicio.

H4: La percepción de valor (valor) tiene una relación positiva con la intención de permanecer y recomendar el servicio (intención).

\section{Método}

\section{Participantes}

Con el objetivo de validar el modelo y las hipótesis se analizaron las percepciones del servicio de inscripciones por Internet (SII) en la licenciatura en Administración, del Instituto Tecnológico Autónomo de México, situado en la Ciudad de México. Se aplicaron 453 cuestionarios directos efectivos a usuarios del sir. Se utilizó un muestreo aleatorio por conglomerados ( 24 grupos de estudiantes en la muestra de un total de 90), donde se aplicó el cuestionario a la totalidad de individuos en cada uno de los grupos seleccionados. De los 453 sujetos muestreados, 176 conforman la muestra utilizada en los modelos de contingencia (modelos 4 y 5 , cuadro 1 ).

\section{Medición}

Como base del instrumento de medición se utilizaron los cuestionarios E-SQual y el E-RecSQual, descritos anteriormente. Se realizó una traducción de los reactivos al español, se analizó cada uno para asegurar que fuera relevante para el servicio y se adaptaron al contexto de las inscripciones por Internet. La dimensión que en el ERecSQual considera como indemnización fue modificada por la dimensión corrección de errores. También se agregaron dos medidas de calidad global para formar 
un total de tres. Se utilizó una escala de 1 a 5 en todos los reactivos y se ordenaron aleatoriamente. Se realizó una prueba piloto para verificar sus adaptaciones.

\section{Análisis}

El análisis realizado se basó en el modelado a través de ecuaciones estructurales (Structural Equation Modeling, SEM). SEM es una técnica de análisis multivariado de tipo confirmatorio, basado en las técnicas de covarianza, regresión y análisis factorial, que permite corroborar relaciones simultáneas entre variables. Esta técnica requiere establecer, previamente, relaciones causales entre variables con base en modelos teóricos (Hair et al., 2010), considerando el criterio de parsimonia (Kline, 2005).

Cabe destacar que existen una serie de problemas potenciales en la utilización de la SEM. Estos problemas pueden surgir en cada una de sus cuatro etapas de aplicación: especificación, datos, análisis e interpretación. La especificación se basó en modelos teóricos de calidad en el servicio. En los datos, una de las principales limitaciones de SEM es el tamaño de muestra por utilizar en la estimación del modelo. Para el modelo normal se tiene una muestra grande $(\mathrm{N}>200)$ y para el modelo de contingencia se tiene una muestra mediana $(100<\mathrm{N}<200)$, lo cual se considera aceptable de acuerdo con el criterio de relación de tamaño de muestra con respecto al número de parámetros por estimar (Kline, 2005).

Debido a la complejidad de los modelos planteados, se calcularon índices promediando las variables observadas de cada factor, lo cual permite simplificar su identificación (Romano et al., 2000; Kline, 2005). El análisis se realizó utilizando el paquete computacional $\mathrm{R}$ y el editor Tinn-R. En el Anexo 3 se presenta el cuadro de estadísticos básicos de las variables. En la presente investigación no se pretende evaluar el servicio comparando las medias de las variables consideradas (Domenge, 2009), el objetivo es identificar los factores de la calidad percibida y sus impactos en la intención de uso y recomendación. El método utilizado para estimar los parámetros del modelo fue máxima verosimilitud, siguiendo el modelaje de una etapa (Kline, 2005) debido al buen ajuste de los modelos y a la consistencia de sus coeficientes estimados. 


\section{Resultados}

\section{Fiabilidad y validez}

Con el objetivo de evaluar la fiabilidad de las variables se utilizó el alfa de Cronbach (Peterson, 1994; Hair et al., 2010), ver cuadro 2. Para las variables de la percepción del servicio normal, global, valor, gloval e intención los valores del alfa se encontraron en el rango de fiabilidad aceptable, considerando 0.7 como el mínimo (Nunnally y Bernstein, 1994).

\section{Cuadro 2}

\section{Análisis de fiabilidad de las variables latentes}

\begin{tabular}{l|c|c}
\hline \multicolumn{3}{c}{ Análisis de Fiabilidad } \\
\hline Constructo & Variables consideradas & Alfa de Cronbach \\
\hline Normal & eficiencia, sistema, confiabilidad, privacidad* & 0.811 \\
Contingencia & respuesta, errores, contacto** & 0.637 \\
Global & GLO1, GLO2, GLO3 & 0.829 \\
Valor & VAL1, VAL2, VAL3 & 0.841 \\
Gloval & GLO1, GLO2, GLO3, VAL1, VAL2, VAL3 & 0.897 \\
Intención & INT1, INT2, INT3,INT4, INT5 & 0.896 \\
\hline
\end{tabular}

El alfa para la medición de la percepción del servicio en caso de contingencia la coloca ligeramente por debajo del mínimo definido por Nunnally y Bernstein (1994), sería recomendable revisar en futuras aplicaciones los reactivos de esta escala para incrementar la fiabilidad en su medición.

La validez de criterio o fiabilidad predictiva (Parasuraman et al., 2005) se analizó a través de los coeficientes (cargas factoriales) de los factores con sus variables observadas (índices). Mediante éstos se puede conocer la fuerza de la relación entre ambos. Para los factores que forman la percepción del servicio normal (cuadro 3), la mayor para predecir la valoración del servicio es la eficiencia seguida por la confiabilidad. En el caso de contingencia, la respuesta es la que tiene una mayor fuerza. Los coeficientes de las variables observadas con global y valor, así como con gloval son semejantes, destacando la variable observada GLO2 referente directamente a la eficiencia. Los más altos para intención corresponden a INT3 e INT2, es decir, animar y recomendar su uso. 


\section{Cuadro 3}

\section{Estimaciones de los cinco modelos}

\begin{tabular}{|c|c|c|c|c|c|}
\hline \multicolumn{6}{|c|}{ Resultados de las estimaciones de los cinco modelos } \\
\hline \multirow[b]{2}{*}{ Relación analizada } & \multicolumn{5}{|c|}{ Valor de los estimadores } \\
\hline & Modelo 1 & Modelo 2 & Modelo 3 & Modelo 4 & Modelo 5 \\
\hline Eficiencia $\leftarrow$ Normal & $0.823 * *$ & $0.823 * *$ & $0.822 * *$ & - & - \\
\hline Sistema $\leftarrow$ Normal & $0.761^{*}$ & $0.763 *$ & $0.761 *$ & - & - \\
\hline Confiabilidad $\leftarrow$ Normal & $0.786^{*}$ & $0.790 *$ & $0.791 *$ & - & - \\
\hline Privacidad $\leftarrow$ Normal & $0.552 *$ & $0.541 *$ & $0.543 *$ & - & - \\
\hline Respuesta $\leftarrow$ Contingencia & $0.802 * *$ & - & - & $0.840 * *$ & $0.838 * *$ \\
\hline Errores $\leftarrow$ Contingencia & $0.726^{*}$ & - & - & $0.638 *$ & $0.640^{*}$ \\
\hline Contacto $\leftarrow$ Contingencia & $0.393 *$ & - & - & $0.414 *$ & $0.413 *$ \\
\hline Normal $\leftarrow$ Global & - & $0.923 *$ & - & - & - \\
\hline Contingencia $\leftarrow$ Global & - & - & - & $0.697 *$ & - \\
\hline Global $\leftarrow$ Valor & - & $0.96 *$ & - & $0.968^{*}$ & - \\
\hline Valor $\longleftarrow$ Intención & - & $0.733^{*}$ & - & $0.765^{*}$ & - \\
\hline Normal $\leftarrow$ Gloval & $1.081^{*}$ & - & $0.919 *$ & - & - \\
\hline Contingencia $\leftarrow$ Gloval & -0.181 & - & - & - & $0.708 *$ \\
\hline Gloval $\leftarrow$ Intención & $0.732 *$ & - & $0.732 *$ & - & $0.770^{*}$ \\
\hline GLO1 $\leftarrow$ GLOBAL & - & $0.692 * *$ & - & $0.619 * *$ & - \\
\hline $\mathrm{GLO} 2 \leftarrow \mathrm{GLOBAL}$ & - & $0.841 *$ & - & $0.840 *$ & - \\
\hline GLO3 $\leftarrow$ GLOBAL & - & $0.813^{*}$ & - & $0.753^{*}$ & - \\
\hline VAL1 $\leftarrow$ VALOR & - & $0.836^{* *}$ & - & $0.795^{* *}$ & - \\
\hline VAL2 $\leftarrow$ VALOR & - & $0.720 *$ & - & $0.679 *$ & - \\
\hline VAL3 $\leftarrow$ VALOR & - & $0.783 *$ & - & $0.780 *$ & - \\
\hline GLO1 $\leftarrow$ GLOVAL & $0.684 * *$ & - & $0.686 * *$ & - & $0.613 * *$ \\
\hline GLO2 $\leftarrow$ GLOVAL & $0.833^{*}$ & - & $0.834 *$ & - & $0.827 *$ \\
\hline GLO3 $\leftarrow$ GLOVAL & $0.806^{*}$ & - & $0.807 *$ & - & $0.740^{*}$ \\
\hline VAL1 $\leftarrow$ GLOVAL & $0.823 * *$ & - & $0.823 * *$ & - & $0.787 *$ \\
\hline VAL2 $\leftarrow$ GLOVAL & $0.711 *$ & - & $0.708 *$ & - & $0.670 *$ \\
\hline VAL3 $\leftarrow$ GLOVAL & $0.769 *$ & - & $0.768 *$ & - & $0.771^{*}$ \\
\hline INT1 $\leftarrow$ INTENCIÓN & $0.745 * *$ & $0.745 * *$ & $0.745 * *$ & $0.748 * *$ & $0.748 * *$ \\
\hline INT2 2 INTENCIÓN & $0.902 *$ & $0.902 *$ & $0.902 *$ & $0.870^{*}$ & $0.870^{*}$ \\
\hline INT3 $\leftarrow$ INTENCIÓN & $0.915^{*}$ & $0.915 *$ & $0.915^{*}$ & $0.893^{*}$ & $0.893^{*}$ \\
\hline INT4 $\leftarrow$ INTENCIÓN & $0.679 *$ & $0.679 *$ & $0.679 *$ & $0.672 *$ & $0.672 *$ \\
\hline INT5 $\leftarrow$ INTENCIÓN & $0.750 *$ & $0.750 *$ & $0.750 *$ & $0.756^{*}$ & $0.756^{*}$ \\
\hline
\end{tabular}

“-” : la relación no se evalúa en ese modelo. * Significativo en p<0.01.

** Se fijó el valor en 1 para realizar la identificación del modelo.

\section{Ajuste del modelo}

Se utilizaron distintos indicadores con el objetivo de evaluar el ajuste de los modelos, (cuadro 4). Se presentan la $\chi^{2}$ y la razón $\chi^{2} / \mathrm{gl}$ como pruebas iniciales de ajuste 
global. Todos los modelos cumplen con el criterio de aceptación de Bollen (1989) y Kline (2005). El criterio de aceptación es para valores menores a tres y hasta un máximo de cinco. Otro indicador de ajuste, RMSEA (Root Mean Square Error of Approximation), se encuentra en el rango aceptable de 0.05 y 0.08 (Millsap, 2002; Kline, 2005; Hair et al., 2010).

\section{Cuadro 4 \\ Medidas de ajuste de los cinco modelos considerados}

\begin{tabular}{c|c|c|c|c|c|c|c|c}
\hline \multicolumn{1}{c}{ Medidas de ajuste } \\
\hline Modelo & $\mathrm{N}$ & $\mathrm{X}^{2}$ & $\begin{array}{c}\text { Gr. de } \\
\text { libertad }\end{array}$ & $\mathrm{X}^{2} / \mathrm{gl}$ & RMSEA & GFI & AGFI & CFI \\
\hline Modelo 1 & 453 & 410.92 & 131 & 3.14 & 0.0687 & 0.904 & 0.875 & 0.945 \\
Modelo 2 & 453 & 236.54 & 87 & 2.72 & 0.0617 & 0.930 & 0.903 & 0.966 \\
Modelo 3 & 453 & 245.72 & 88 & 2.79 & 0.0629 & 0.927 & 0.901 & 0.964 \\
Modelo 4 & 176 & 139.98 & 74 & 1.89 & 0.0714 & 0.894 & 0.84963 & 0.9490 \\
Modelo 5 & 176 & 141.27 & 75 & 1.88 & 0.0710 & 0.893 & 0.84984 & 0.9488 \\
\hline
\end{tabular}

Con respecto al índice GFI (Goodness of Fit Index), los modelos tienen un ajuste aceptable, aunque los modelos que miden exclusivamente las dimensiones de la percepción de contingencia no tienen un ajuste como los de la percepción normal. Se presenta también el AGFI (Adjusted Goodness of Fit Index), que es una extensión del GFI ajustado por el cociente de grados de libertad (Hair et al., 2010). Se considera que un valor aceptable para el AGFI se encuentra alrededor de 0.9, como en los modelos normal.

El índice CFI de Bentler -el cual no se ve afectado por el tamaño de muestra (Bentler, 1990) y es muy estable cuando se tiene un número grande de variables en el modelo (Chau y Hocevar, 1995) — indica que se cuenta con un ajuste adecuado. $\mathrm{Al}$ analizar globalmente las medidas de ajuste para los todos los modelos se identifica que el GFI, el AGFI y el CFI muestran que el modelo con mejor ajuste es el modelo 2 .

Los resultados del análisis validan las hipótesis de acuerdo con los siguientes criterios:

H1: Se encuentra sustento para esta hipótesis porque la relación normal $\rightarrow$ global presenta coeficientes positivos $(\mathrm{p}<0.01)$ en todos los modelos analizados que la contienen (modelos 1, 2 y 3 ). 
H2: La percepción del servicio de contingencia sólo fue significativa cuando se analizaba aislada de percepción del servicio normal y en los usuarios que tuvieron problemas con el servicio. Los resultados de los modelos 4 y 5 dan sustento para esta hipótesis, pues presentan coeficientes positivos $(\mathrm{p}<0.01)$.

H3: Los resultados obtenidos proporcionan sustento para esta hipótesis porque los coeficientes para esta relación son positivos $(\mathrm{p}<0.01)$.

H4: La evidencia muestra que sí existe una relación positiva entre la percepción de valor agregado y la intención de permanecer o recomendar el servicio (modelos 2 y $4, \mathrm{p}<0.01)$.

Las hipótesis plantean que los factores que forman la percepción de la calidad en servicios por Internet puros se comportan de acuerdo con la especificación del modelo (Figura 1), así como los modelos complementarios, donde la calidad percibida se conforma por dos procesos, dependiendo de si el usuario experimenta o no problemas con el servicio. De esta manera, los usuarios evalúan los factores de la percepción normal y éstas tienen una relación positiva con la percepción de calidad global y valor. Por otra parte, cuando el usuario experimenta problemas, adicionalmente evalúa los factores de la percepción de contingencia, que tienen una relación positiva con la calidad global, el valor y la intención.

Por otro lado, los factores con relaciones más altas que determinan la variable normal son la eficiencia y la confiabilidad en todos los modelos que la consideran (1,2 y 3 , cuadro 3). Para la variable contingencia, la relación más alta es con respuesta (modelos 1, 4 y 5). Estos resultados sugieren que efectivamente, los usuarios están orientados principalmente a la obtención de resultados y no tanto a otros factores (Zeithaml, 2002 y 2006) como el contacto personal en servicios tradicionales.

\section{Conclusiones y recomendaciones}

Los factores de la percepción normal de la calidad, como los de la percepción de contingencia son relevantes para la formación de la percepción global de calidad y valor. Existe evidencia que muestra que los usuarios del servicio evalúan la eficiencia del sitio y la confiabilidad del servicio en primera instancia, seguidas de la consecución de las promesas de servicio sobre la disponibilidad de servicios y el respeto a la privacidad. No obstante, cuando se experimentan problemas con el servicio, además de evaluar los factores anteriores, también se evalúa la capacidad 
de respuesta ante el problema, la posibilidad de corregir errores y la existencia de contacto personal para solucionar problemas.

Estos resultados sugieren que los principales factores que los prestadores de servicio deben tomar como base para el diseño y rediseño de un sistema de servicios por Internet son la eficiencia y la confiabilidad debido a que son las más importantes en la formación de la percepción de calidad y valor en el servicio. Se puede apreciar que estos dos factores conforman la dimensión de funcionalidad del sistema, objetivo básico del usuario de sI.

Los proveedores de si deberán considerar que existen factores que están fuera de su control y que pueden afectar esta dimensión como, por ejemplo, la velocidad de conexión y las características de la computadora (hardware) y de la aplicación utilizada (software). Los prestadores de servicios deben ser conscientes que el valor percibido del uso de un si es resultado de la percepción global de calidad y que el valor percibido es precursor de la intención de permanecer y/o recomendar el sistema, por lo que dicha calidad percibida puede tener un impacto importante en la tasa de reutilización y en la futura recomendación verbal de persona a persona del servicio.

Dos elementos adicionales que pueden resultar significativos en la percepción de la calidad y el logro de los objetivos del usuario es su experiencia tanto en el uso de servicios por Internet como en el servicio particular que se esté utilizando. A mayor experiencia y a mayor respaldo institucional el usuario probablemente tendría un acceso más eficiente y confiable, requiriendo de un menor uso de factores de contingencia.

Sería recomendable comparar modelos de servicios por Internet en los que existieran sustitutos para el logro de los objetivos del usuario, pues en el servicio estudiado no existen opciones directas. Adicionalmente, sería interesante realizar análisis en servicios que tengan un costo asociado a su uso para poder evaluar el efecto del precio en el valor percibido. 


\section{Referencias}

Aaker, D. y R. Bagozzi (1979). Unobservable Variables In Structural Equation Models with an Application in Industrial Selling. Journal of Marketing Research. Mayo. 16, 147-58.

Bentler, P. M. (1990). Comparative Fit Indexes in Structural Models. Psychological Bulletin. 107, 238-246.

Bollen, K. (1989). Structural Equations with Latent Variables. USA: Wiley.

Bolton, R. N. y J. H. Drew (1991). A Multistage Modelo of Customers' Assesments of Service Quality and Value. Journal of Consumer Research. 55(1), $1-9$.

Brady, M. K. y J.J. Cronin (2001). Some New Thoughts on Conceptualizing Perceived Service Quality: A Hierarchical Approach. Journal of Marketing. Julio. 65, 34-49.

Chau, H. y D. Hocevar (1995). The Effects of Number of Measured Variables on Goodness-of-Fit in Confirmatory FactorAnalysis. Disponible en: http://www. eric.ed.gov/ERICWebPortal/custom/portlets/recordDetails/detailmini.jsp? nfbp $=$ true \& \&ERICExtSearchSearchValue 0=ED387516\&EROCExtSearch SearchType $0=$ eric accno\&accno=ED387516

Cronin, J. y S.Taylor (1994). SERVPERF vs SERVQUAL: Reconciling Performance-based and Perceptions-minus-expectations Measurement of Service Quality. The Journal of Marketig. Enero. 58, 125-131.

Domenge, R. (2009). Importancia de los factores de transferencia de contenido en una escuela de negocios: percepciones de estudiantes y profesores. Cuadernos de Estudios Empresariales. Vol. 19. Madrid: Universidad Complutense de Madrid.

Fransi, E. (2003). Conceptualización de la Calidad de Servicio al Cliente percibida en el Comercio Electrónico. Evaluación y Aplicación en el Establecimiento Virtual. Tesis Doctoral. Universidad de Lleda. 
Grönroos, C. (1982). Strategic Management and Marketing in Service Sector. Cambridge, MA: Marketing Science Institute.

Hair, J. R., W. Black, B. J Babin y R. E. Anderson (2010). Multivariate data Analysis. 7a. ed., USA: Prentice Hall

Hawkins, Del I, R. J. Best y K. A. Coney (2004). Consumer Behavior: building marketing strategy. 9a. ed., Boston, MA. USA: McGraw-Hill Irwin.

Kang, G. y J. James (2004). Service Quality Dimensions: an examination of Grönroos's service quality model. Managing Service Quality. 14(4), 266-277.

Kline, R.B. (2005). Structural Equations Modeling. Segunda edición. USA: The Guilford Press.

Lehtinen, U. y J. R. Lehtinen, (1982). Service Quality: A Study of Quality Dimensions. Service Management Institute. Finlandia OY.

Loiacono, E., R.Watson y D. Goodhue (2002). WebQual ${ }^{t m}$ : A Web Site Quality Instrument. American Marketing Association: Winter Marketing Educators' Conference, Austin, Texas. 432-438.

Millsap, R. (2002). Structural equation modeling: A user's guide. En Drasgow, F. y Schmitt, N. (Editores). Measuring and Analyzing Behavior in Organizations: Advances in measurement and data analysis. San Francisco, USA: Jossey-Bass. 257-301.

Nunnally, J. y I. Bernstein (1994). Psychometric Theory. New York, USA: McGraw-Hill.

Parasuraman, A., V.A. Zeithaml y L. Berry, (1985). A Conceptual Model of Service Quality and Its Implications for Future Research. Journal of Marketing. 49(4), 41-50.

y A. Malhotra (2005). E-S-QUAL. A Multiple-Item Scale for Assessing Electronic Service Quality. Journal of Service Research. 7(3), 213-233. 
Peterson, R. (1994). A Meta-analysis of Cronbach's Coefficient Alpha. The Journal of Consumer Research. 21(2), 381-391.

Romano, C., G. Tanewski y P. Smyrnios (2000). Capital Structure Decision Making: A Model for Family Business. Journal of Business Venturing. 16, 285310.

Ruyter, K., J. Bloemer y P. Peeters (1997). Merging Service Quality and Service Satisfaction: An empirical test of an integrative model. Journal of Economic Psychology. 18, 387-406.

Sasser, W.E., R. P. Olsen y D. D. Wyckoff (1972). Management of Service Operations. USA: Allyn and Bacon, Inc.

Swan, J.E. y L. J. Combs (1976). Product Performance and Consumer Satisfaction: A New Concept. Journal of Marketing. Abril. 40, 25-33.

Zeithaml, V.A., M. J. Bitner y D. D. Gremler (2006). Services Marketing: Integrating Customer Focus across the Firm. 4a., ed., USA: McGraw Hill. understanding e-service quality: implications for future research and managerial practice. Working Paper: Marketing Science Institute. Reporte No. 00-115.

(2002). Service Quality Delivery through Web Sites: A Critical Review of Extant Knowledge. Journal of the Academy of Marketing Science. 30(4), 362-375. 


\title{
Anexo 1
}

\section{Variables latentes y variables observadas}

\author{
VL: EFICIENCIA, la facilidad y velocidad de acceder y usar un sitio. \\ VO: EFF1, encontrar información; EFF2, facilidad navegación; EFF3, rapidez transacción; EFF4, \\ organización; EFF5, velocidad descarga.
}

VL: SISTEMA, la consecución de las promesas de servicio sobre la disponibilidad de productos o servicios. VO: SYS1, disponibilidad; SYS2, apertura correcta; SYS3, mensajes error; SYS4, funcionamiento adecuado.

VL: CONFIABILIDAD, el correcto funcionamiento del sitio a nivel técnico.

VO: FUL1, accesibilidad; FUL2, tiempo transacción; FUL3, rapidez del registro; FUL4, registro correctamente; FUL6, veracidad información.

VL: PRIVACIDAD, el resguardo de la información privada del cliente.

VO: PRI1, confianza; PRI2, privacidad; PRI3, protección.

VL: NORMAL, la percepción de calidad general.

Constructo (Aaker et al, 1979) que agrupa todos los factores que son considerados como relevantes en los usuarios del servicio (EFICIENCIA, SISTEMA, CONFIABILIDAD y PRIVACIDAD).

VL: RESPUESTA, la capacidad de respuesta, manejo efectivo de problemas en el servicio.

VO: RES1, realizar modificaciones; RES3, realizar correcciones; RES4, indicaciones; RES5, rapidez.

VL: ERRORES, corrección de errores, la posibilidad de realizar cambios en respuesta a contingencias. VO: COM1, posibilidad correcciones; COM2, acceso y cambios; COM3, equivocación y cambios.

VL: CONTACTO, la existencia de asistencia por teléfono o por Internet.

VO: CON1, teléfono; CON2, existencia; CON3, facilidad.

VL: CONTINGENCIA, la percepción de calidad en caso de problemas.

Se agrupan los factores que son relevantes cuando existe algún problema con el servicio (RESPUESTA, ERRORES y CONTACTO).

VL: GLOBAL, calidad global percibida

VO: GLO1, calidad; GLO2, eficiencia; GLO3, eficacia.

VL: VALOR, valor percibido.

VO: VAL1, conveniencia (facilidad, comodidad); VAL2, control proceso; VAL3, valor agregado.

VL: GLOVAL, percepción de calidad y valor. Se conjuntaron las VO de GLOBAL y de VALOR como consecuencia de un análisis de factores exploratorio que muestra que estas variables están altamente relacionadas.

VL: INTENCIÓN, intenciones de permanecer / recomendar el servicio por el usuario.

VO: INT1, decir; INT2, recomendar; INT3, animar a otros a usarlo; INT4, considerarlo primera opción; INT5, volver.

Notas: VL: variable latente. VO: variables observadas.

Las variables en negritas son las que tienen un coeficiente mayor con respecto a su correspondiente VL. 


\section{Anexo 2}

\section{Especificación de los modelos}

\begin{tabular}{|c|c|}
\hline $\begin{array}{c}\text { Modelo } \\
\text { global } \\
\text { (Figura 1) }\end{array}$ & 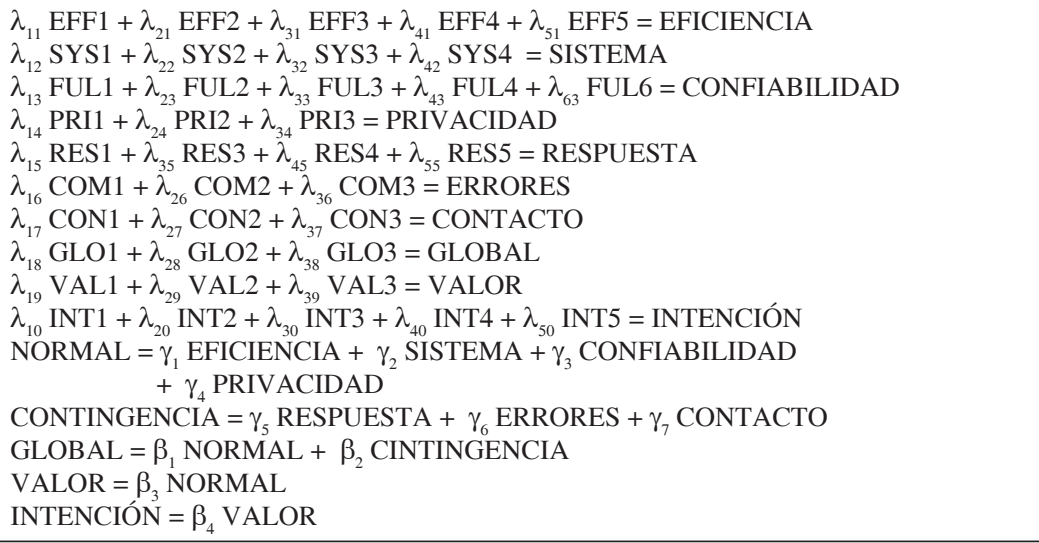 \\
\hline Modelo 1 & $\begin{array}{l}\lambda_{1} \text { EFICIENCIA }+\lambda_{2} \text { SISTEMA }+\lambda_{3} \text { CONFIABILIDAD }+\lambda_{4} \text { PRIVACIDAD }=\text { NORMAL } \\
\lambda_{5} \text { RESPUESTA }+\lambda_{6} \text { ERRORES }+\lambda_{7} \text { CONTACTO }=\text { CONTINGENCIA } \\
\lambda_{18} \text { GLO } 1+\lambda_{28} \text { GLO } 2+\lambda_{38} \text { GLO3 }+\lambda_{19} \text { VAL } 1+\lambda_{29} \text { VAL } 2+\lambda_{39} \text { VAL } 3=\text { GLOVAL } \\
\lambda_{10} \text { INT } 1+\lambda_{20} \text { INT2 }+\lambda_{30} \text { INT3 }+\lambda_{40} \text { INT } 4+\lambda_{50} \text { INT5 }=\text { INTENCIÓN } \\
\text { GLOVAL }=\beta_{1} \text { NORMAL }+\beta_{2} \text { CINTINGENCIA } \\
\text { INTENCIÓN }=\beta_{3} \text { GLOVAL }\end{array}$ \\
\hline Modelo 2 & $\begin{array}{l}\lambda_{1} \text { EFICIENCIA }+\lambda_{2} \text { SISTEMA }+\lambda_{3} \text { CONFIABILIDAD }+\lambda_{4} \text { PRIVACIDAD }=\text { NORMAL } \\
\lambda_{18} \text { GLO } 1+\lambda_{28} \text { GLO } 2+\lambda_{38} \text { GLO3 }=\text { GLOBAL } \\
\lambda_{19} \text { VAL } 1+\lambda_{29} \text { VAL } 2+\lambda_{39} \text { VAL3 }=\text { VALOR } \\
\lambda_{10} \text { INT } 1+\lambda_{20} \text { INT } 2+\lambda_{30} \text { INT3 } 3+\lambda_{40} \text { INT4 }+\lambda_{50} \text { INT5 }=\text { INTENCIÓN } \\
\text { GLOBAL }=\beta_{1} \text { NORMAL } \\
\text { VALOR }=\beta_{2} \text { GLOBAL } \\
\text { INTENCIÓN }=\beta_{3} \text { VALOR }\end{array}$ \\
\hline Modelo 3 & $\begin{array}{l}\lambda_{1} \text { EFICIENCIA }+\lambda_{2} \text { SISTEMA }+\lambda_{3} \text { CONFIABILIDAD }+\lambda_{4} \text { PRIVACIDAD }=\text { NORMAL } \\
\lambda_{18} \text { GLO } 1+\lambda_{28} \text { GLO } 2+\lambda_{38} \text { GLO } 3+\lambda_{19} \text { VAL } 1+\lambda_{29} \text { VAL } 2+\lambda_{39} \text { VAL } 3=\text { GLOVAL } \\
\lambda_{10} \text { INT } 1+\lambda_{20} \text { INT } 2+\lambda_{30} \text { INT } 3+\lambda_{40} \text { INT } 4+\lambda_{50} \text { INT5 }=\text { INTENCIÓN } \\
\text { GLOVAL }=\beta_{1} \text { NORMAL } \\
\text { INTENCIÓN }=\beta_{3} \text { GLOVAL }\end{array}$ \\
\hline Modelo 4 & $\begin{array}{l}\lambda_{1} \text { RESPUESTA }+\lambda_{2} \text { ERRORES }+\lambda_{3} \text { CONTACTO }=\text { CONTINGENCIA } \\
\lambda_{18} \text { GLO } 1+\lambda_{28} \text { GLO } 2+\lambda_{38} \text { GLO3 }=\text { GLOBAL } \\
\lambda_{19} \text { VAL } 1+\lambda_{29} \text { VAL } 2+\lambda_{39} \text { VAL } 3=\text { VALOR } \\
\lambda_{10} \text { INT } 1+\lambda_{20} \text { INT } 2+\lambda_{30} \text { INT3 }+\lambda_{40} \text { INT } 4+\lambda_{50} \text { INT5 }=\text { INTENCIÓN } \\
\text { GLOBAL }=\beta_{1} \text { CONTINGENCIA } \\
\text { VALOR }=\beta_{2} \text { GLOBAL } \\
\text { INTENCIÓN }=\beta_{3} \text { VALOR }\end{array}$ \\
\hline Modelo 5 & $\begin{array}{l}\lambda_{1} \text { RESPUESTA }+\lambda_{2} \text { ERRORES }+\lambda_{3} \text { CONTACTO }=\text { CONTINGENCIA } \\
\lambda_{18} \text { GLO } 1+\lambda_{28} \text { GLO } 2+\lambda_{38} \text { GLO } 3+\lambda_{19} \text { VAL } 1+\lambda_{29} \text { VAL } 2+\lambda_{39} \text { VAL3 } 3=\text { GLOVAL } \\
\lambda_{10} \text { INT } 1+\lambda_{20} \text { INT2 } 2+\lambda_{30} \text { INT } 3+\lambda_{40} \text { INT } 4+\lambda_{50} \text { INT } 5=\text { INTENCIÓN } \\
\text { GLOVAL }=\beta_{1} \text { CONTINGENCIA } \\
\text { INTENCIOON }=\beta_{3} \text { GLOVAL }\end{array}$ \\
\hline
\end{tabular}




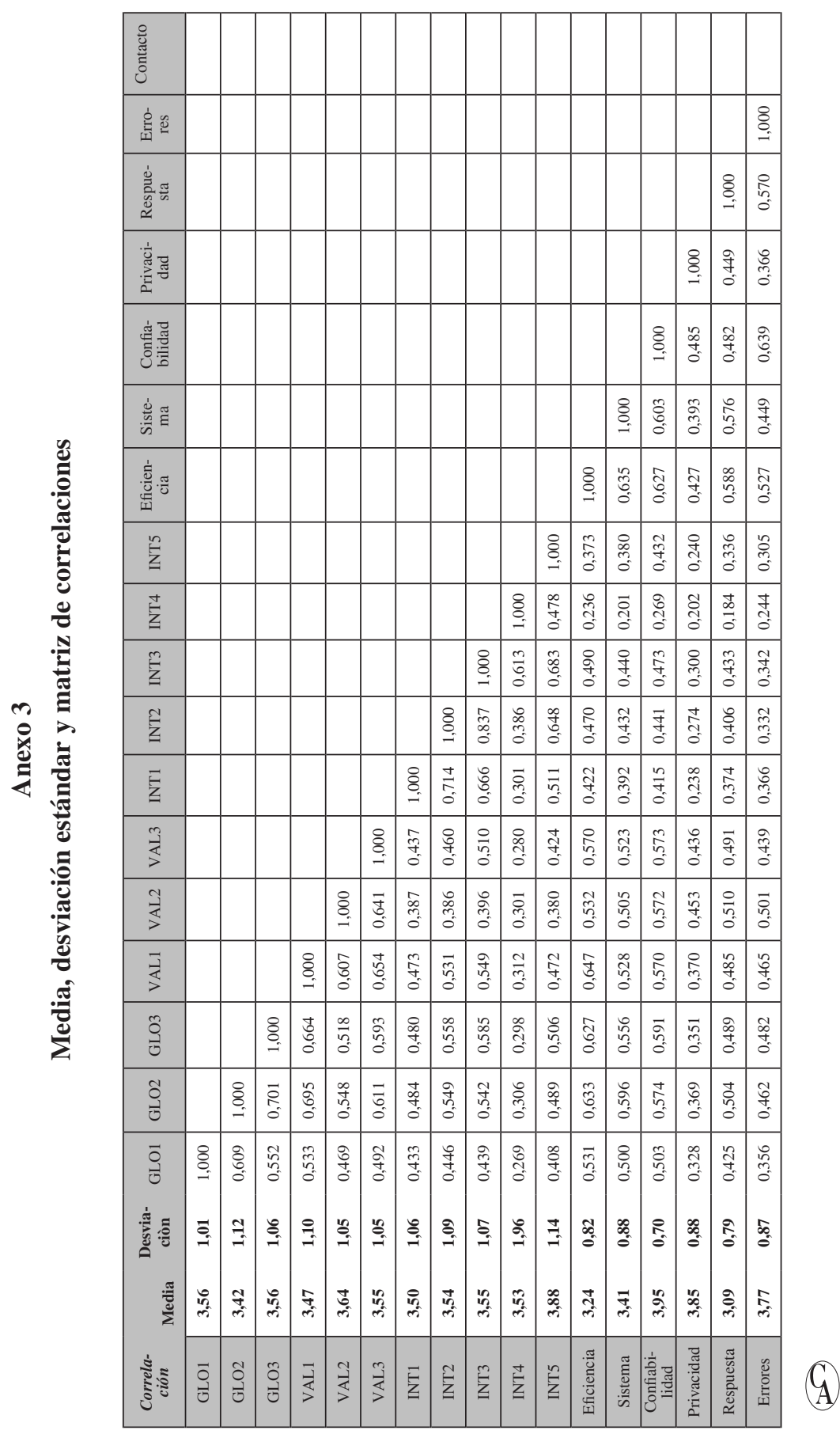

Contaduría y Administración, No. 233, enero-abril 2011: 11-31 